United Nations Educational Scientific and Cultural Organization and International Atomic Energy Agency

INTERNATIONAL CENTRE FOR THEORETICAL PHYSICS

\title{
ENERGY-LEVEL STATISTICS \\ AND TIME RELAXATION IN QUANTUM SYSTEMS
}

\author{
J.L. Gruver \\ International Centre for Theoretical Physics, Trieste, Italy \\ and
}

Grupo de Sistemas Dinámicos, Centro Regional Norte, Universidad de Buenos Aires, Casilla de Correo 2, 1638 Vicente López, Buenos Aires, Argentina,

\section{J. Aliaga}

Departamento de Física, Facultad de Ciencias Exactas y Naturales, Universidad de Buenos Aires,

Pabellón I, Ciudad Universitaria, 1428 Buenos Aires, Argentina,

Hilda A. Cerdeira

International Centre for Theoretical Physics, Trieste, Italy,

Pier A. Mello

Instituto de Física, Universidad Nacional Autónoma de México, Apartado 20-364, 01000 México

and

A.N. Proto

Grupo de Sistemas Dinámicos, Centro Regional Norte, Universidad de Buenos Aires, Casilla de Correo 2, 1638 Vicente López, Buenos Aires, Argentina.

MIRAMARE - TRIESTE

May 1997 


\begin{abstract}
We study a quantum-mechanical system, prepared, at $t=0$, in a model state, that subsequently decays into a sea of other states whose energy levels form a discrete spectrum with given statistical properties. An important quantity is the survival probability $P(t)$, defined as the probability, at time $t$, to find the system in the original model state. Our main purpose is to analyze the influence of the discreteness and statistical properties of the spectrum on the behavior of $P(t)$. Since $P(t)$ itself is a statistical quantity, we restrict our attention to its ensemble average $\langle P(t)\rangle$, which is calculated analytically using randommatrix techniques, within certain approximations discussed in the text. We find, for $\langle P(t)\rangle$, an exponential decay, followed by a revival, governed by the two-point structure function of the statistical spectrum, thus giving a nonzero asymptotic value for large $t$ 's. The analytic result compares well with a number of computer simulations, over a time range discussed in the text.
\end{abstract}




\section{INTRODUCTION}

The problem of finding the time relaxation of a quantum-mechanical system after it has been prepared in some model state is of great interest in many fields of physics. Perhaps one of the oldest examples goes back to the analysis by Weisskopf and Wigner [1] of a decaying state. The main features of the derivation can be obtained in the following schematic model. The state $|\lambda\rangle$ is coupled at $t=0$ to an infinite discrete spectrum of equally spaced levels $|n\rangle$, $\Delta$ being the level spacing, via the matrix elements $v_{n}=\langle i|v| n\rangle$, assumed to be independent of $n: v_{n}=v$. Then, taking the continuum limit $\Delta \rightarrow 0(|n\rangle \rightarrow|E\rangle)$, subject to the condition $2 \pi v^{2} / \Delta=\Gamma$ (where $\Gamma$ is a constant), the survival probability, i.e., the probability of finding the system in state $|\lambda\rangle$ at time $t$, is found to have the familiar exponential behavior $P(t)=e^{-\Gamma t}$ (in units in which $\left.\hbar \equiv 1\right)$.

The above schematic model can be taken as the paradigm for a number of physical applications. Consider, for instance, the quasiparticle concept in many-body theory [2]. In this case, the model state $|\lambda\rangle$ can be taken to represent a single-particle state, which is coupled to the remaining states via the two-body interaction. For a bound system, the resulting width is sometimes known as the "spreading width." Actually, various Green function techniques have been developed to treat this problem [2]. Still in the framework of many-body theory, one can put in the above language the important problem of describing the resonances generated by the decay of "bound states embedded in the continuum" via the two-body interaction [3]. Actually, the general problem of resonance decay in scattering theory and the deviations from the pure exponential decay have been of interest to several authors over the years: see, e.g., Ref. [4], and the references cited therein. Another problem that fits the above scheme and was of interest a few decades ago in the realm of nuclear physics is the decay, via the Coulomb interaction, of a state of good isospin embedded in a sea of states of a different isospin [5], and the related spreading width.

In the case of a bound system, the spectrum the model state decays into is discrete; in the approaches indicated above, the influence, in the relaxation process, of the discreteness 
of the spectrum or its statistical properties was not contemplated. In contrast, Ref. [6] and some of the references cited there consider the relaxation of a harmonic oscillator coupled to a bath of $N$ harmonic oscillators with equally spaced frequencies ( $\Delta$ being the spacing), without taking the continuum limit. In the $N \rightarrow \infty$ limit an exponential decay is found, with a revival at $\tau_{r} \approx 2 \pi / \Delta$ and multiples thereof. Reference [7] discusses the dynamics of an electron inside a chaotic quantum dot (for a review of quantum dots see Ref. [8]): the analysis considers the time evolution of the probability to find the particle at a point $\mathbf{r}_{2}$ if it was located at $\mathbf{r}_{1}$ at $t=0$. Using supersymmetry methods, it is found that quantum interference, the discreteness of the energy spectrum, and the corresponding level statistics lead to a nontrivial time-dependent behavior governed by the Fourier transform of the twolevel correlation function $[9,10]$ (see also Refs. $[11,12]$ ). A similar statement is made, without proof, in Ref. [13]: there, a wave packet is constructed at $t=0$ as a linear combination of $N$ states - contained in a stretch of energy $\delta E$, the mean spacing being $\Delta$ - obeying one of the random-matrix statistics $[9,10]$; it is stated that the ensemble-averaged survival probability is governed by the Fourier transform of the two-level correlation function, in agreement with Ref. [7].

The purpose of the present paper is to analyze the influence of the discreteness and statistical properties of the spectrum in the survival probability $P(t)$ of a model state. The analysis is performed in a language entirely similar to that described at the beginning of this Introduction in connection with the Weisskopf-Wigner model, but using a random-matrix spectrum instead of an equally spaced one and without ever taking the continuum limit. The ensemble average $\langle P(t)\rangle$ is calculated using random-matrix techniques: although the calculation is performed within certain approximations to be defined below, we found the simplicity of the derivation appealing.

In the next section we define the model and solve the corresponding time-independent Schrödinger equation. The survival probability and its ensemble average are calculated in Sec. III, where a comparison with numerical simulations is also presented. 


\section{THE MODEL. THE TIME-INDEPENDENT SOLUTION}

In the Introduction we described a number of physical systems in which the relaxation of a model state $|\lambda\rangle$ into a "sea" of levels $|\mu\rangle$ via a residual interaction is of great interest. In this section we propose a model for this problem and analyze the solution of the corresponding time-independent Schrödinger equation. The time-dependent solution -the topic of main interest in this paper- will be studied in the next section.

The states $|\lambda\rangle$ and $|\mu\rangle$ are assumed to be solutions of an unperturbed Hamiltonian $H_{0}$,

$$
\begin{aligned}
& \left(E_{\lambda}-H_{0}\right)|\lambda\rangle=0, \\
& \left(E_{\mu}-H_{0}\right)|\mu\rangle=0,
\end{aligned}
$$

$E_{\lambda}$ being embedded in the sea of levels $E_{\mu}$. The total Hamiltonian is

$$
H=H_{0}+v,
$$

where the residual interaction $v$ only couples the state $|\lambda\rangle$ with the states $|\mu\rangle$; i.e., its only nonzero matrix elements are

$$
\langle\lambda|v| \mu\rangle=v_{\lambda \mu}
$$

Some of the properties of the solutions of the complete Schrödinger equation

$$
\left(E_{i}-H\right)|i\rangle=0
$$

can be obtained in an elementary way, as discussed in Refs. [5,14] and briefly indicated in what follows. Expanding the eigenstates $|i\rangle$ in terms of $|\lambda\rangle$ and $|\mu\rangle$ as

$$
|i\rangle=\frac{|\lambda\rangle+\sum_{\mu} c_{\mu}^{(i)}|\mu\rangle}{\sqrt{1+\sum_{\mu}\left|c_{\mu}\right|^{2}}},
$$

we can write Eq. (4) as the system of coupled equations

$$
\begin{aligned}
E_{\lambda}+\sum_{\mu} v_{\lambda \mu} c_{\mu} & =E, \\
v_{\mu \lambda}+E_{\mu} c_{\mu} & =E c_{\mu},
\end{aligned}
$$


whose solutions are the eigenvalues $E_{i}$ and the eigenvector components $c_{\mu}^{(i)}$. We find, for $c_{\mu}$,

$$
c_{\mu}=\frac{v_{\mu \lambda}}{E-E_{\mu}}
$$

which can thus be eliminated from Eqs. (6) to give, for $E$, the dispersionlike relation

$$
\sum_{\mu} \frac{\left|v_{\lambda \mu}\right|^{2}}{E_{\mu}-E}=E_{\lambda}-E .
$$

A plot of the two sides of Eq. (8) as a function of $E$ gives the solutions $E_{i}$ as the abscissas of the intersections. We can see that there is a solution between every two successive $E_{\mu}$ 's, and one smaller and one larger than all the $E_{\mu}$ 's. The solutions $E_{i}$ approach $E_{\mu}$ as we go ever farther away from $E_{\lambda}$.

The "strength function," i.e., the probability of finding the model state $|\lambda\rangle$ in the exact eigenstate $|i\rangle$, is given, from Eqs. (5) and (7), by

$$
w_{i} \equiv|\langle\lambda \mid i\rangle|^{2}=\frac{1}{1+\sum_{\mu}\left[v_{\lambda \mu} /\left(E_{i}-E_{\mu}\right)\right]^{2}} .
$$

We shall see in the next section [see Eq. (30)] that the strength function plays an important role in the calculation of the time-dependent survival probability; we thus devote the rest of this section to studying some of its properties.

Since the state $|\lambda\rangle$ is normalized, the $w_{i}$ 's obey the sum rule

$$
\sum_{i} w_{i}=1
$$

Far away from $E_{\lambda}$, where $E_{i} \approx E_{\mu}$, Eq. (8) allows writing $w_{i}$ as

$$
w_{i} \approx \frac{\left|v_{\lambda \mu}\right|^{2}}{\left(E_{\lambda}-E_{i}\right)^{2}},
$$

in agreement with perturbation theory.

It is interesting that for a "picket-fence" (PF) model, in which the $E_{\mu}$ 's are equally spaced -their distance being $\Delta$ - and infinite in number, and all the $\left|v_{\lambda \mu}\right|^{2}=v^{2}$, the strength function can be found exactly as

$$
w_{i}^{\mathrm{PF}}=\frac{v^{2}}{\left(E_{i}-E_{\lambda}\right)^{2}+(\Gamma / 2)^{2}},
$$


i.e., a Lorentzian of width

$$
\Gamma=\frac{2 \pi v^{2}}{\Delta}\left[1+\left(\frac{\Delta}{\pi v}\right)^{2}\right]^{1 / 2},
$$

which in turn goes over, in the continuum limit, to the result mentioned in the Introduction.

In the model with which are we concerned, the energy levels $E_{\mu}$ are assumed to obey some statistical distribution with constant average spacing $\Delta$ and to be infinite in number; we suppose, for simplicity, that all the $\left|v_{\lambda \mu}\right|^{2}=v^{2}$. The strength function $w_{i}$ will then depend not only on $E_{i}$, as for the PF, Eq. (12), but also on all the other $E_{j}$ 's. This last dependence is negligible far from $E_{\lambda}$, as Eq. (11) shows, but this may not be the case in other regions. We represent $w_{i}$ as

$$
w_{i}=w\left(E_{i}\right)+\delta w_{i}
$$

Here, $w\left(E_{i}\right)$ is a continuous function of its argument that represents, for fixed $E_{i}$, an average over all the other levels; $\delta w_{i}$ represents the fluctuations around that average and thus averages to zero for fixed $E_{i}$ (its full ensemble average thus vanishes too: $\left\langle\delta w_{i}\right\rangle=0$ ). The sum rule (10) reads

$$
\sum_{i} w\left(E_{i}\right)+\sum_{i} \delta w_{i}=1
$$

The first term, i.e.,

$$
W=\sum_{i} w\left(E_{i}\right)
$$

is a statistical quantity, because the $E_{i}$ 's fluctuate from sample to sample: it is actually a linear statistic, in the language of Dyson and Mehta [15]. Thus $W$ fluctuates around

$$
\langle W\rangle=1
$$

[the magnitude of the fluctuations will be found below, in Eq. (25)]; but $W$, added to the second term in Eq. (15), always gives 1. Since the $E_{i}$ 's occur symmetrically in the distribution function, the probability density $p\left(E_{i}\right)$ for a single eigenvalue is identical for all $i$ 's. For $N$ levels in an interval $L, p\left(E_{i}\right)=1 / L$ and Eq. (17) gives 


$$
1=N\left\langle w\left(E_{1}\right)\right\rangle=N \int_{L} w(E) \frac{d E}{L} .
$$

Letting $N, L \rightarrow \infty$, with $L / N=\Delta$, we find

$$
\frac{1}{\Delta} \int w(E) d E=1
$$

Inspired by the behavior of $W_{i}$ far from $E_{\lambda}$ [see Eq. (11)] and the result for the PF Eq. (12), we propose, a simple model for $w\left(E_{i}\right)$, the Lorentzian

$$
w\left(E_{i}\right)=\frac{\Gamma \Delta / 2 \pi}{\left(E_{i}-E_{\lambda}\right)^{2}+(\Gamma / 2)^{2}}
$$

that has been normalized so as to satisfy Eq. (19).

We now turn to the fluctuations of $W$. For a spectrum with "stationary," i.e., energyindependent, statistical properties, Ref. [15] finds, for the variance of a linear statistic like $W$

$$
\operatorname{var}(W)=\frac{1}{\Delta} \int_{-\infty}^{+\infty}|\phi(\tau)|^{2}[1-b(\Delta \tau)] d \tau
$$

Here,

$$
\phi(\tau)=\int_{-\infty}^{+\infty} w(E) e^{-2 \pi i E \tau} d E
$$

and $b(k)$ is the two-level form factor, defined as the Fourier transform of the two-point cluster function [9]. We shall assume that the $E_{\mu}$ 's form a spectrum with stationary statistical properties and that these properties are not significantly altered in the spectrum of the $E_{i}^{\prime}$ 's: this is an approximation, partially verified in Ref. [16], whose relaxation would require further analysis. In particular, for a Gaussian orthogonal ensemble (GOE) [9], $b(k)$ is given by

$$
b(k)=\left\{\begin{array}{rr}
1-2|k|+|k| \ln (1+2|k|), & |k| \leq 1 \\
-1+|k| \ln \left[\frac{2|k|+1}{2|k|-1}\right], & |k| \geq 1 .
\end{array}\right.
$$

If we adopt, for $w(E)$, the Lorentzian model $(20)$, then 


$$
\phi(\tau)=\Delta e^{-\pi \Gamma|\tau|}
$$

If $\Gamma / \Delta \gg 1$, we may approximate $b(k)$ in Eq. (21) for small values of the argument $(\approx$ $1-2|k|)$, so that

$$
\operatorname{var}(W) \approx 2 \int_{-\infty}^{+\infty}|\phi(\tau)|^{2}|\tau| d \tau=\left(\frac{\Delta}{\pi \Gamma}\right)^{2}
$$

On the other hand, squaring the expression

$$
W=1-\sum_{i} \delta w_{i}
$$

[see Eqs. (15) and (16)] and ensemble averaging, we obtain, for the variance of $W$,

$$
\operatorname{var}(W)=\sum_{i, j}\left\langle\delta w_{i} \delta w_{j}\right\rangle
$$

Comparing with Eq. (25) we thus find the requirement

$$
\sum_{i, j}\left\langle\delta w_{i} \delta w_{j}\right\rangle=\left(\frac{\Delta}{\pi \Gamma}\right)^{2},
$$

that the fluctuating quantities $\delta w_{i}$ must fulfill.

\section{THE RELAXATION OF THE MODEL STATE}

Suppose that at $t=0$ we prepare the system described in the preceding section in the model state $|\lambda\rangle$. As a function of time, the system will be found in the state

$$
|\psi(t)\rangle=\sum_{i}|i\rangle\langle i \mid \lambda\rangle e^{-i E_{i} t}
$$

(we use units in which $\hbar \equiv 1$ ).

The survival probability amplitude for finding the system, at time $t$, in the original state $|\lambda\rangle$ is

$$
\alpha(t) \equiv\langle\lambda \mid \psi(t)\rangle=\sum_{i} w_{i} e^{-i E_{i} t}
$$


which is thus expressed in terms of the strength function of the preceding section, Eq. (9). For an ensemble of spectra, as we considered in the preceding section, $\alpha(t)$ is a random quantity; its ensemble average is given by

$$
\begin{aligned}
\langle\alpha(t)\rangle & =\sum_{i}\left\langle\left[w\left(E_{i}\right)+\delta w_{i}\right] e^{-i E_{i} t}\right\rangle=\sum_{i}\left\langle w\left(E_{i}\right) e^{-i E_{i} t}\right\rangle \\
& =N \int w\left(E_{1}\right) e^{-i E_{1} t} p\left(E_{1}\right) d E_{1}=N \int_{L} w(E) e^{-i E t} \frac{d E}{L} \\
& \rightarrow \frac{1}{\Delta} \int w(E) e^{-i E t} d E,
\end{aligned}
$$

in the same limit as in preceding section, right below Eq. (18). If we model $w(E)$ as in Eq. (20), choosing $E_{\lambda}=0$, we find, for the ensemble-averaged survival probability amplitude, the exponential decay law

$$
\langle\alpha(t)\rangle=e^{-\Gamma t / 2}
$$

We now turn to the main topic of the present paper: the study of the survival probability

$$
P(t)=|\alpha(t)|^{2}
$$

which, from Eqs. (30) and (14), can be written as

$$
\begin{aligned}
P(t) & =\sum_{i, j} w_{i} w_{j} e^{i\left(E_{j}-E_{i}\right) t} \\
& =\sum_{i, j}\left[w\left(E_{i}\right)+\delta w_{i}\right]\left[w\left(E_{j}\right)+\delta w_{j}\right] e^{i\left(E_{j}-E_{i}\right) t} .
\end{aligned}
$$

Of course, the full statistical distribution of the random quantity $P(t)$ for fixed $t$ is of interest; here we shall confine ourselves to the calculation of its first moment. From Eq. (34) we can write

$$
\langle P(t)\rangle=\langle P(t)\rangle^{(0)}+Q(t),
$$

where

$$
\langle P(t)\rangle^{(0)}=\left\langle|\widehat{\alpha}(t)|^{2}\right\rangle,
$$

with 


$$
\hat{\alpha}(t)=\sum_{i} w\left(E_{i}\right) e^{-i E_{i} t} \equiv \sum_{i} w_{t}\left(E_{i}\right)
$$

and

$$
\begin{aligned}
Q(t)= & \sum_{i, j}\left\langle\left[w\left(E_{i}\right)\left(\delta w_{j}\right)+\left(\delta w_{i}\right) w\left(E_{j}\right)\right] e^{i\left(E_{j}-E_{i}\right) t}\right\rangle \\
& +\sum_{i, j}\left\langle\left(\delta w_{i}\right)\left(\delta w_{j}\right)^{i\left(E_{j}-E_{i}\right) t}\right\rangle .
\end{aligned}
$$

The first term in Eq. (35), i.e., $\langle P(t)\rangle^{(0)}$, can be calculated if we realize that the quantity $\widehat{\alpha}(t)$ is again a linear statistic: this we do below [Eq. (41)]. We have not been able to evaluate the term $Q(t)$; however, we show that this term is quite small for not too large $t$ and verify this statement numerically in cases where $\Gamma \gg \Delta$. For large $t$ we also show that its average over a long time interval is positive and verify this statement numerically as well (see Sec. III A below).

The analysis of Ref. [15], which was already applied to write Eq. (21), now leads to

$$
\left\langle\widehat{\alpha}(t) \widehat{\alpha}^{*}(t)\right\rangle-\langle\hat{\alpha}(t)\rangle\left\langle\hat{\alpha}^{*}(t)\right\rangle=\frac{1}{\Delta} \int_{-\infty}^{+\infty}\left|\phi_{t}(\tau)\right|^{2}[1-b(\Delta \tau)] d \tau
$$

where $b(k)$ is again the two-level form factor and

$$
\begin{aligned}
\phi_{t}(\tau) & =\int_{-\infty}^{+\infty} w_{t}(E) e^{-2 \pi i E \tau} d E \\
& =\int_{-\infty}^{+\infty} w(E) e^{-2 \pi i E(\tau+t / 2 \pi)} d E
\end{aligned}
$$

which reduces to $\phi(\tau)$ of Eq. (22) when $t=0$. Equations. (31), (36), and (39) then yield

$$
\langle P(t)\rangle^{(0)}=\left|\phi_{t}(0)\right|^{2} / \Delta^{2}+\frac{1}{\Delta} \int_{-\infty}^{+\infty}\left|\phi_{t}(\tau)\right|^{2}[1-b(\Delta \tau)] d \tau
$$

For the Lorentzian model $(20)$ for $w(E)$, we have

$$
\phi_{t}(\tau)=\Delta e^{-\pi \Gamma|\tau+t / 2 \pi|} .
$$

Equation (41) is the main result of this paper: it gives $\langle P(t)\rangle^{(0)}$ as a quadrature of known functions. For $\Gamma \gg \Delta,\langle P(t)\rangle^{(0)}$ describes an exponential decay, which is then followed by a "revival," governed by the two-level form factor. Equation (41) can be approximated by 


$$
\langle P(t)\rangle^{(0)} \approx\langle P(t)\rangle^{(1)} \equiv e^{-\Gamma t}+\frac{\Delta}{\pi \Gamma}\left[1-b\left(\frac{\Delta t}{2 \pi}\right)\right]
$$

for $\Gamma \gg \Delta$. We first apply Eq. (41) for $t=0$. Using Eqs. (19), (21), (25), and (40) we find

$$
\langle P(0)\rangle^{(0)}=1+\left(\frac{\Delta}{\pi \Gamma}\right)^{2}
$$

if $\Gamma \gg \Delta$. For $Q(0)$, Eq. (38) gives

$$
\begin{aligned}
Q(0) & =2\left\langle\left[\sum_{i} w\left(E_{i}\right)\right]\left[\sum_{j} \delta w_{j}\right]\right\rangle+\sum_{i, j}\left\langle\left(\delta w_{i}\right)\left(\delta w_{j}\right)\right\rangle \\
& =2\left\langle\left(1-\sum_{i} \delta w_{i}\right)\left[\sum_{j} \delta w_{j}\right]\right\rangle+\sum_{i, j}\left\langle\left(\delta w_{i}\right)\left(\delta w_{j}\right)\right\rangle \\
& =-\sum_{i, j}\left\langle\left(\delta w_{i}\right)\left(\delta w_{j}\right)\right\rangle=-\left(\frac{\Delta}{\pi \Gamma}\right)^{2} .
\end{aligned}
$$

Here we used Eq. (28). Results (44) and (45) add up to 1, as they should. If $\Gamma \gg \Delta$, Eq. (45) shows that $Q(0)$ is second order in the small quantity $\Delta / \pi \Gamma$; we thus expect, for not too large times, $\langle P(t)\rangle^{(0)}$ to dominate over $Q(t)$.

Consider now the average of $\langle P(t)\rangle$ over an infinite time interval, that we denote with a bar. From Eq. (35) we find

$$
\overline{\langle P(t)\rangle}=\overline{\langle P(t)\rangle^{(0)}}+\overline{Q(t)}
$$

Using Eqs. (36) and (37), we can express the first term as

$$
\overline{\langle P(t)\rangle^{(0)}}=\left\langle\sum_{i}\left[w\left(E_{i}\right)\right]^{2}\right\rangle=\frac{1}{\Delta} \int[w(E)]^{2} d E,
$$

which, evaluated with the Lorentzian model (20), gives

$$
\overline{\langle P(t)\rangle^{(0)}}=\frac{\Delta}{\pi \Gamma}
$$

which should then also be the asymptotic value of $\langle P(t)\rangle^{(0)}$ for large times. This result has a very appealing interpretation. The probability of finding the eigenstate $|i\rangle$ of $H$ in the vector state $|\psi(t)\rangle$ is $w_{i}$, independent of time [see Eq. (29)]; $w_{i}$ is concentrated in a region of width $\Gamma\left[\right.$ Eq. (20)], where there are $\sim n=\Gamma / \Delta$ levels, so that $\left\langle w_{i}\right\rangle \sim 1 / n$ inside that region. 
From the standpoint of the eigenstates $|\lambda\rangle,|\mu\rangle$ of $H_{0}$, the probability is concentrated, at $t=0$, in the model state $|\lambda\rangle$ and in none of the states $|\mu\rangle$; as $t \rightarrow \infty$, according to Eq. (48), it is as if the probability had been spread among $\sim n$ neighboring levels.

We can estimate the second term in Eq. (46) by time averaging Eq. (38) and approximating $\left\langle w\left(E_{i}\right)\left(\delta w_{i}\right)\right\rangle \approx 0$, so that $\overline{Q(t)} \approx \sum_{i}\left\langle\left(\delta w_{i}\right)^{2}\right\rangle$. This indicates that the correction on $\overline{\langle P(t)\rangle^{(0)}}$ provided by $\overline{Q(t)}$ is positive. This correction can be further estimated as follows. Call $x_{i}=\langle\lambda \mid i\rangle$, so that $w_{i}=x_{i}^{2}$. As in the above paragraph, we recall that the energy span over which $w_{i}$ is appreciable is $\sim \Gamma$ and contains $\sim n=\Gamma / \Delta$ levels. Suppose that the $x_{i}$ are Gaussian variables with zero centroid and the same variance $V$. Then $\left\langle x_{i}^{2}\right\rangle=\left\langle w_{i}\right\rangle=V$ and $\left\langle x_{i}^{4}\right\rangle=\left\langle w_{i}^{2}\right\rangle=3 V^{2}$, so that $\operatorname{var}\left(w_{i}\right)=\left\langle w_{i}^{2}\right\rangle-\left\langle w_{i}\right\rangle^{2}=2 V^{2}$. From Eq. (10), $V=1 / n$, so that $\overline{Q(t)} \sim n \operatorname{var}\left(w_{i}\right) \sim 1 / n \sim \Delta / \Gamma$. This estimate indicates that for large times $Q(t)$ is no longer negligible compared with $\langle P(t)\rangle^{(0)}$ and that the two quantities could be of the same order of magnitude.

\section{A. Numerical simulations}

We present a scheme that is useful for the numerical calculation of the survival probability $P(t)$ of Eq. (33). We can expand the wave function of the system at time $t[\mathrm{Eq} .(29)]$ in terms of the model state $|\lambda\rangle$ and the basis $|\mu\rangle$ as

$$
|\psi(t)\rangle=\alpha(t)|\lambda\rangle+\sum_{\mu} \alpha_{\mu}(t)|\mu\rangle
$$

The coefficients $\alpha(t)$ and $\alpha_{\mu}(t)$ satisfy the set of ordinary differential equations

$$
\begin{aligned}
\frac{d \alpha(t)}{d t} & =-i E_{\lambda} \alpha(t)-i \sum_{\mu} v_{\lambda \mu} \alpha_{\mu}(t), \\
\frac{d \alpha_{\mu}(t)}{d t} & =-i E_{\mu} \alpha_{\mu}(t)-i v_{\lambda \mu}^{*} \alpha(t) .
\end{aligned}
$$

Using Eqs. (50) we find the set of $(N+1)^{2}$ ordinary differential equations

$$
\frac{d P(t)}{d t}=-\sum_{\mu=1}^{N} F_{\mu}(t),
$$




$$
\begin{aligned}
\frac{d P_{\mu}(t)}{d t} & =F_{\mu}, \\
\frac{d F_{\mu}(t)}{d t} & =-\left(E_{\lambda}-E_{\mu}\right) I_{\mu}(t)+2\left|v_{\lambda \mu}\right|^{2} P(t)-\sum_{\nu=1}^{N} \mathcal{I}_{\mu \nu}(t), \\
\frac{d I_{\mu}(t)}{d t} & =\left(E_{\lambda}-E_{\mu}\right) F_{\mu}(t)-\sum_{\nu=1}^{N} \mathcal{F}_{\mu \nu}(t), \\
\frac{d \mathcal{F}_{\mu \nu}(t)}{d t} & =\left(E_{\nu}-E_{\mu}\right) \mathcal{I}_{\mu \nu}(t)-\left|v_{\lambda \mu}\right|^{2} I_{\nu}(t)+\left|v_{\lambda \nu}\right|^{2} I_{\mu}(t), \\
\frac{d \mathcal{I}_{\mu \nu}(t)}{d t} & =-\left(E_{\nu}-E_{\mu}\right) \mathcal{F}_{\mu \nu}(t)+\left|v_{\lambda \mu}\right|^{2} F_{\nu}(t)+\left|v_{\lambda \nu}\right|^{2} F_{\mu},
\end{aligned}
$$

from which we can find the time evolution of $P(t)$. In Eqs. (51) we have defined

$$
\begin{aligned}
P_{\mu}(t) & \equiv\left|\alpha_{\mu}(t)\right|^{2}, \\
F_{\mu}(t) & \equiv i\left[v_{\lambda \mu} \alpha^{*}(t) \alpha_{\mu}(t)-v_{\lambda \mu}^{*} \alpha_{\mu}^{*}(t) \alpha(t)\right], \\
I_{\mu}(t) & \equiv v_{\lambda \mu} \alpha^{*}(t) \alpha_{\mu}(t)+v_{\lambda \mu}^{*} \alpha_{\mu}^{*}(t) \alpha(t), \\
\mathcal{F}_{\mu \nu}(t) & \equiv i\left[v_{\lambda \mu}^{*} v_{\lambda \nu} \alpha_{\mu}^{*}(t) \alpha_{\nu}(t)-v_{\lambda \mu} v_{\lambda \nu}^{*} \alpha_{\nu}^{*}(t) \alpha_{\mu}\right], \\
\mathcal{I}_{\mu \nu}(t) & \equiv v_{\lambda \mu}^{*} v_{\lambda \nu} \alpha_{\mu}^{*}(t) \alpha_{\nu}(t)+v_{\lambda \mu} v_{\lambda \nu}^{*} \alpha_{\nu}^{*}(t) \alpha_{\mu}(t),
\end{aligned}
$$

with $\mu, \nu=1, \ldots, N$. The system of equations (51) is formally equivalent to the one obtained in Ref. [6] and the magnitudes defined by Eqs. (52) can be thought of as a particular case of the "relevant" operators introduced also in that reference. A number of numerical simulations were performed, in order to verify the approximations under which the above analytical results were obtained. For the $E_{\mu}$ 's an unfolded (i.e., with uniform density) GOE spectrum with $N=800$ levels centered at $E_{\lambda}$ was used. The coupling matrix element, in units of the mean spacing $\Delta$, was chosen as $v / \Delta=2.08$, giving, for the width of the strength function, in the same units, $\Gamma / \Delta=2 \pi v^{2} / \Delta^{2}=27.27$. The results of the computer simulations are given in Figure 1, which shows $P(t)$ averaged over an ensemble of 100 members; the error bars indicate the fluctuations arising from the finite size of the ensemble. The dash-dotted line is a plot of the theoretical expression $\langle P(t)\rangle^{(1)}$, Eq. (43); no significant difference was found with $\langle P(t)\rangle^{(0)}$, obtained carrying out numerically the integration indicated in Eq. (41). In the abscissa, the time is indicated in units of the 
"Heisenberg time" $1 / \Delta$. We observe that, up to times $t \sim 2.4 / \Delta \sim 65 / \Gamma$ (see inset), $\langle P(t)\rangle^{(1)}$ gives a good quantitative description of the data. The reason for the difference, or discrepancy, in the vicinity of the minimum is not very clear; numerically we have not found a clear indication of saturation in this difference with the number of levels, which was increased up to $N=800$. There is some evidence [17] that such a discrepancy might be connected with the difference in statistical properties between the spectrum of the $E_{i}$ 's and that of the $E_{\mu}$ 's; if this is the case, using, in Eq. (43), the $b(\Delta t / 2 \pi)$ appropriate for the levels $E_{\mu}$ should improve the agreement. Also, should there be any evidence for a deviation with respect to the Lorentzian model of the strength function, we could modify the $\phi_{t}(\tau)$ of Eq. (42) accordingly. For larger times the numerical results are larger than the analytical ones, in agreement with the discussion given above. The qualitative trend, i.e., an exponential decay followed by a nonzero asymptotic value, is similar to the one found in Ref. [12]; this asymptotic behavior is similar to that indicated in Ref. [11] and to the "echo" observed in Ref. [7].

\section{SUMMARY}

In this paper we consider a quantum-mechanical system, prepared, at $t=0$, in a model state, that subsequently decays into a sea of other states whose energy levels form a discrete spectrum with an average level spacing $\Delta$. The main purpose of the paper is to analyze the influence of the discreteness and statistical properties of the spectrum in the time dependence of the survival probability $P(t)$. The ensemble average $\langle P(t)\rangle$ of the survival probability is given in Eq. (35). The first term, $\langle P(t)\rangle^{(0)}$, is calculated explicitly in Eq. (41), under the assumption that the stationary (i.e., energy-independent) statistical properties of the original spectrum are not significantly altered in the perturbed spectrum. For $\Gamma \gg \Delta$, $\langle P(t)\rangle^{(0)}$ of Eq. (41) does not differ significantly from its approximation $\langle P(t)\rangle^{(1)}$ of Eq. (43). Its general trend, for $\Gamma \gg \Delta$, is an exponential decay $e^{-\Gamma t}$, followed by a revival, governed by the two-point structure function. We have not been able to evaluate the second 
term, $Q(t)$; however, for not too large times we found it to be quadratic in the small quantity $\Delta / \Gamma$, so that $\langle P(t)\rangle^{(1)}$ alone describes well (except, to a certain extent, for the vicinity of the minimum, as discussed above) the results of computer simulations up to times on the order of 2.4 times the Heisenberg time $1 / \Delta$, or $\sim 65$ decay times $1 / \Gamma$.

\section{ACKNOWLEDGMENTS}

J.L.G. and J.A. acknowledge support from CONICET, Argentina. H.A.C. acknowledges support from the Istituto Nazionale de Fisica Nucleare (INFN). A.N.P. acknowledges support from the Comisión de Investigaciones Científicas de la Provincia de Buenos Aires (CIC). J.L.G. and P.A.M. are grateful for support and hospitality of the International Centre for Theoretical Physics (ICTP), Trieste, Italy. P.A.M. wishes to thank H. Hernández, J. Flores, and T.H. Seligman for important discussions on the validity of the assumptions inherent in the theoretical analysis. 


\section{REFERENCES}

[1] V. Weisskopf and E. P. Wigner, Z. Phys. 63, 54 (1930).

[2] D. Pines, The Many-Body Problem (W. A. Benjamin, Inc., New York, 1962), p. 31.

[3] C. Mahaux and W. A. Weidenmüller, Shell Model Approach to Nuclear Reactions (North-Holland Publishing Company, Amsterdam, 1969); D. Agassi, H. A. Weidenmüller, and G. Mantzouranis, Phys. Rep. 3, 145 (1975).

[4] G. García Calderón, G. Loyola, and M. Moshinsky, in Symmetries in Physics, edited by A. Frank and K. B. Wolf (Springer-Verlag, Berlin, 1992), p. 273; G. García Calderón, J. L. Mateos, and M. Moshinsky, Phys. Rev. Lett. 74, 337 (1995).

[5] E. P. Wigner, in Isobaric Spin in Nuclear Physics, edited by J. D. Fox and D. Robson (Academic Press, New York, 1966), p. 437.

[6] J. L. Gruver, J. Aliaga, Hilda A. Cerdeira, and A. N. Proto, Phys. Rev. E 51, 6263 (1995).

[7] V. N. Prigodin, B. L. Altshuler, K. B. Efetov, and S. Iida, Phys. Rev. Lett. 72, 546 (1994).

[8] C. W. J. Beenakker and H. van Houton, in Solid State Physics: Advances in Research and Applications, edited by H. Ehrenreich and D. Turnbull (Academic Press, New York, 1991), Vol. 44, pp. 1-228.

[9] M. L. Mehta, Random Matrices and the Statistical Theory of Energy Levels (Academic Press, New York, 1991).

[10] T. A. Brody, J. Flores, J. B. French, P. A. Mello, A. Pandey, and S. S. M. Wong, Rev. Mod. Phys. 53, 385 (1981).

[11] F. Leyvraz, J. Quezada, T. H. Seligman, and M. Lombardi, Phys. Rev. Lett. 67, 2921 (1991). 
[12] H. Hernández, Tesis Profesional, Facultad de Ciencias, Universidad Nacional Autónoma de México (U.N.A.M.), México, 1995.

[13] P. A. Mello, in Mesoscopic Quantum Physics, edited by E. Akkermans, G. Montambaux, J-L Pichard, and J. Zinn-Justin (North-Holland, Amsterdam, 1995), p. 454.

[14] E. Braun and S. V. Godoy, Physica 86A, 377 (1977); E. Braun and P. A. Mello, Physica A 143, 547 (1987).

[15] F. J. Dyson and M. L. Mehta, J. Math. Phys. 4, 701 (1963).

[16] T. A. Brody, P. A. Mello, J. Flores, and O. Bohigas, Lett. Nuovo Cimento 7, 707 (1973).

[17] H. Hernández, J. Flores, and T. H. Seligman (private communication). 


\section{FIGURES}

FIG. 1. The time evolution of the average survival probability obtained from a numerical simulation is represented as the solid line: an unfolded GOE spectrum with 800 levels centered at $E_{\lambda}$ was used, with $v / \Delta=2.08$ and $\Gamma / \Delta=27.27$; an ensemble of 100 elements was constructed. The dash-dotted line represents the result of the analytical study $\langle P(t)\rangle^{(1)}$. The inset shows the evolution for shorter times, where the error bars due to the finite sample size were included. 
i

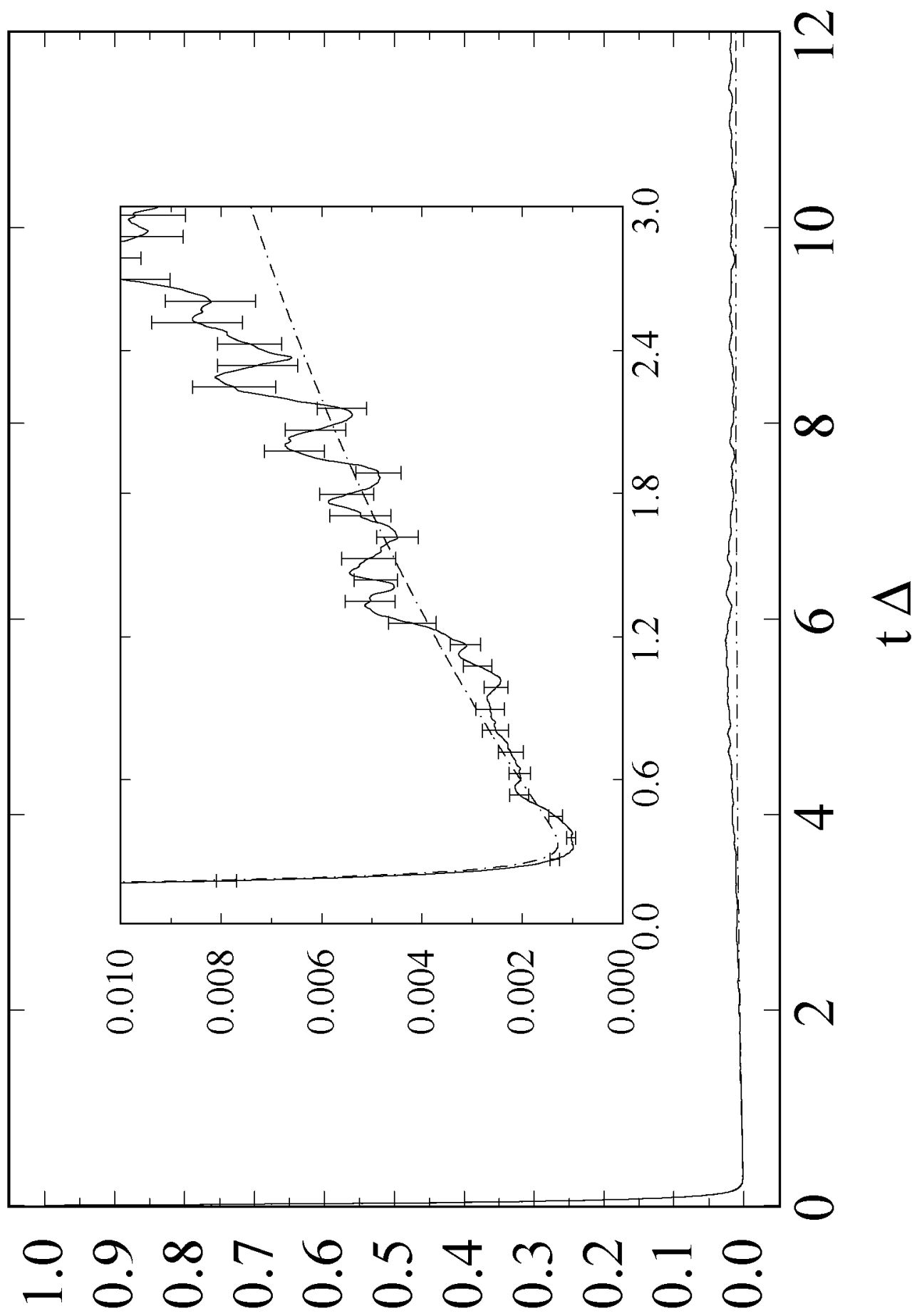

Кұ!!!qeqo. 